\title{
Thermal vacuum tests for the ESA's OPS-SAT mission
}

\author{
Manuel Kubicka $(\mathbb{D}) \cdot$ Reinhard Zeif · Maximilian Henkel · Andreas Johann Hörmer
}

Received: 4 October 2021 / Accepted: 9 November 2021 / Published online: 18 February 2022

(C) The Author(s) 2022

\begin{abstract}
OPS-SAT is an ESA nanosatellite launched in December 2019. The spacecraft is open for thirdparty experiments, which can use almost all functions provided by the spacecraft and take full control of it. Depending on the experiment and usage of the payload, the power consumption of the spacecraft may be as small as a few watts but can exceed $30 \mathrm{~W}$ at full load. The peak power production lies in the same order of magnitude, which is highly demanding for thermal regulation. This article describes the preparation and execution of the OPS-SAT Thermal Vacuum (TVAC) test campaign and discusses the limitations and restrictions that had to be taken into account, such as technical limitations with respect to mounting the spacecraft inside the TVAC chamber. Additionally, the procedure of identifying a comprehensive test scenario is discussed. The general approach of TVAC tests and the results of one full test cycle are presented, and the key findings are discussed. The goal is to address the problems and limitations that were encountered during the TVAC test campaign and to provide some ideas and suggestions for improvement for the future.
\end{abstract}

Keywords OPS-SAT · TVAC · CubeSat · Test campaign

\section{Thermal-Vakuum-Tests für die OPS-SAT-Mission der ESA}

Zusammenfassung OPS-SAT ist ein ESA-Nanosatellit der im Dezember 2019 gestartet wurde. Der Satellit ist offen zugänglich für externe Experimente, welche beinahe die gesamte zur Verfügung stehende

\footnotetext{
M. Kubicka $(\bowtie) \cdot$ R. Zeif · M. Henkel · A. J. Hörmer Institute of Communication Networks and Satellite Communications, Graz University of Technology, Inffeldgasse 12, $8010 \mathrm{Graz}$, Austria manuel.kubicka@tugraz.at
}

Funktionalität nutzen und volle Kontrolle über den Satelliten übernehmen können. Je nach Experiment und Verwendung der Komponenten des Satelliten liegt der Energieverbrauch in der Größenordnung einiger weniger Watt, kann aber unter Volllast mehr als $30 \mathrm{~W}$ betragen. Die maximale Energieproduktion des Satelliten liegt in derselben Größenordnung, was hohe Ansprüche an die thermische Regulierung stellt. Dieser Artikel befasst sich mit der Vorbereitung und Ausführung der OPS-SAT-Thermal-Vakuum(TVAC)Test-Kampagne und beschreibt die Einschränkungen und Limitierungen, die dafür berücksichtigt werden mussten. Auf technischer Seite werden etwa die Limitierungen bezüglich Befestigung und Platzierung des Satelliten innerhalb der TVAC-Kammer beschrieben. Zusätzlich wird die Vorgehensweise zur Identifizierung eines umfassenden Test-Szenarios diskutiert. Die allgemeine Vorgehensweise der TVAC-Tests wird vorgestellt, und die Ergebnisse werden anhand eines vollen Testzyklus gezeigt. Die wichtigsten Erkenntnisse daraus werden ebenfalls präsentiert. Das Ziel ist es, die Probleme und Einschränkungen während der Test-Kampagne zu besprechen und Ideen sowie Verbesserungsvorschläge für die Zukunft aufzuzeigen.

Schlüsselwörter OPS-SAT $\cdot$ TVAC $\cdot$ CubeSat · TestKampagne

\section{Introduction}

OPS-SAT is an ESA 3U Cubesat, built by Graz University of Technology (TUG) and serves the purpose of breaking the "has never flown - will never fly" cycle, by providing a powerful experimentation platform in space [1]. OPS-SAT is open for experiments from universities, industry or private researches, completely free of charge. The spacecraft includes a wide variety of payloads, to account for many different types of 


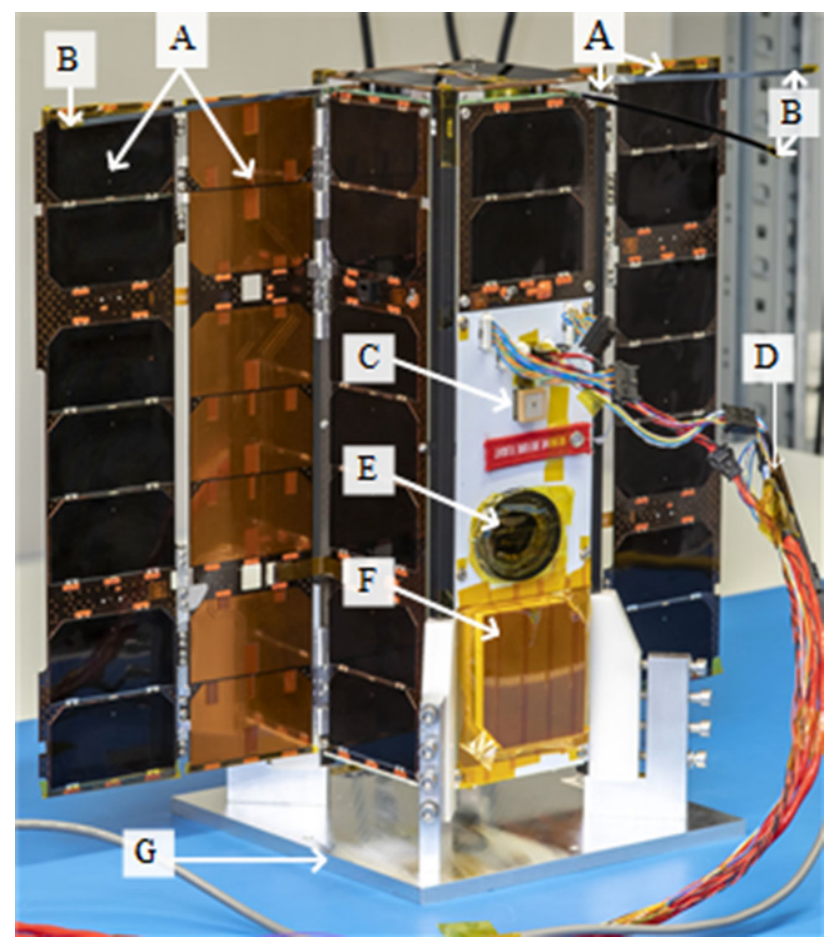

Fig. 1 OPS-SAT is shown on a lab bench. The spacecraft is in post launch configuration, i.e. with deployed solar arrays and UHF antennas. The deployable solar wings are marked with $A$ and the UHF and SDR antennas are marked as $B$. $C$ shows the GPS antenna and $D$ shows the umbilical cord, that is connected for testing and charging. $E$ shows the star tracker baffle and lens, covered by protective tape. $F$ shows the S-band patch antenna and $G$ shows the mechanical stand (not part of the spacecraft)

experiments. It includes UHF, S-Band and X-Band communication systems, a Software Defined Radio (SDR) and a coarse and a fine Attitude Determination and Control System (ADCS) with reaction wheels and a startracker. Further on board are an optical receiver, a HD camera, a GPS module and a retroreflector [2]. On the experiment side, the spacecraft and its payloads are controlled via the so-called Satellite Experimental Processing Platform (SEPP) [7]. The SEPP provides basically full control over the spacecraft, by exposing high and low level interfaces to the experimenter. In case of unforeseen behaviour or any potential risk, the currently active of the two OPS-SAT on-board computers (OBCs) takes over and interrupts the experiment to ensure safety of the spacecraft. An overview of OPS-SAT can be found in Fig. 1, showing the spacecraft in post-launch configuration, with deployed solar arrays and antennas [4].

Payloads Any of the OPS-SAT payloads that are shown in Fig. 2 can be used and controlled by an experiment $[2,4]$. This leads to a large combination of different use cases, each of which with individual requirements in terms of power and resulting thermal behaviour. The nature of purely radiative heat exchange between the spacecraft and the environment,

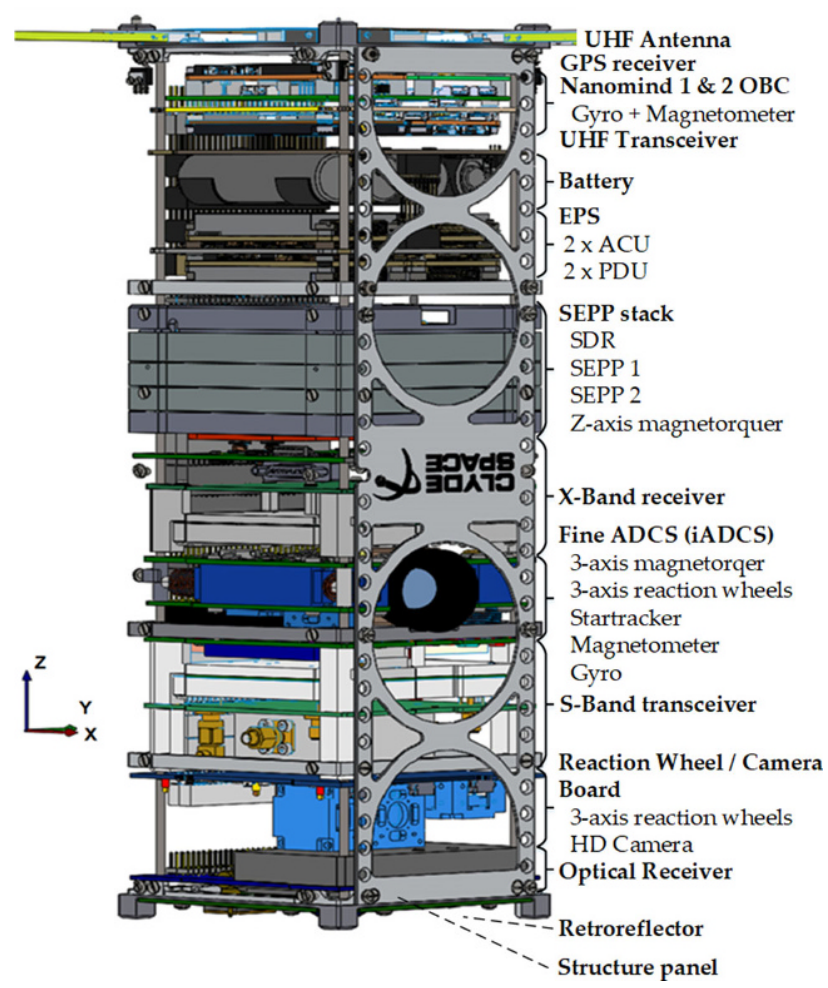

Fig. 2 OPS-SAT bus and payload component location inside the spacecraft structure. The top section of the spacecraft hosts the OBCs, the EPS and battery, GPS and the UHF communication chain. The payloads follow below and take up roughly $2 \mathrm{U}$ of the $3 \mathrm{U}$ CubeSat structure. The Retroreflector is mounted on the bottom panel and is therefore not visible in the image

as it is the case during TVAC tests, leads to extensive test time periods, in order to reach thermal equilibrium states. It is therefore not feasible to account for all possible experimental scenarios on OPS-SAT and a single, representative use case had to be chosen for the TVAC test campaign.

Power consumption A challenging aspect of the spacecraft is the relatively small $3 \mathrm{U}$ form factor, paired with a comparably high power consumption, that can exceed $30 \mathrm{~W}$ in some scenarios. The satellite is equipped with two double deployable solar wings in order to accommodate for its power requirements. The main contributors to power consumption are the SEPP and the S-Band transceiver. Since the SEPP is the basis for most experiments, this unit will be powered on continuously throughout the course of an experiment. Depending on the type of experiment, the SEPP power usage can reach $7-8 \mathrm{~W}$ of continuous power draw. The S-Band adds another $10-12 \mathrm{~W}$ of power draw. This combined power draw of almost two thirds of the total capacity of the OPS-SAT Electrical Power Supply (PSU) leaves little headroom for all the other payloads. The S-Band transmitter, however, is only powered during ground station contact and cannot be powered on for more than 15 min continu- 
ously due to thermal constraints. While the combined power limits of the spacecraft have to account for the S-Band transmitter, it's thermal influence can be neglected, as is evident by on-orbit telemetry (TM) [5].

TVAC tests The OPS-SAT TVAC test campaign has been carried out at the facilities of RUAG Space in Vienna. The goal is to determine the reliability and functionality of all spacecraft components throughout the widest possible temperature range under vacuum conditions and as close to in orbit conditions as possible. An additional goal of the tests is to determine the thermal dependency between individual components, as well as the temperature relations between them. The OPS-SAT battery was chosen as the so-called Temperature Reference Point (TRP), since it is both crucial for mission operations and one of the most thermally sensitive components. The TVAC tests consist of several phases that include powered and passive states of the spacecraft, in order to approach the respective operational and non-operational temperature limits of the components. More details on the individual phases can be found in Sect. 2.3. The results show a close correlation between the temperature of the SEPP and the battery, as well as temperature gradient from the SEPP towards the outer edges of the spacecraft, leading to the conclusion that the SEPP is a strongly contributing factor to the overall thermal behaviour of the spacecraft.

\section{OPS-SAT thermal vacuum tests}

The following section highlights the key considerations for the OPS-SAT thermal vacuum tests. The scope of this article only allows for a very condensed summary of such a test campaign and it is aimed to highlight some key points of the general approach, phases of the tests and a summary of limitations and restrictions that were encountered during the tests and during preparation. First, due to the versatility of OPS-SAT experiments, a representative use in terms of active payloads had to be defined. In terms of power generation, the lack of availability of a sun simulator means that charging power is limited by the umbilical connection. Further, the radio transmitters for UHF and S-Band could not be activated during the tests, in order to avoid damaging the corresponding receiver units due to high power reflections. This leads to a decreased overall power consumption. Finally, the time constraints of the test campaign only allowed for one full test cycle.

\section{Test scenario}

Representative use case OPS-SAT allows for a wide range of experiments that are accompanied with individual configurations of payloads. As such, each configuration has its individual power draw and is accompanied by corresponding heat dissipation and distribution throughout the spacecraft. As it is not

\begin{tabular}{l|l|l|l|l|l|l}
\hline \multicolumn{1}{l}{ Table 1} & OPS-SAT TVAC test: payloads in use \\
\hline Unit & State & Comment \\
\hline UHF Antenna & On & \\
\hline OBC 1 & On & \\
\hline OBC 2 & Disabled & Cold redundancy \\
\hline UHF transceiver & On & RX only \\
\hline GPS & On & \\
\hline EPS & On & \\
\hline SDR & On & Idle (low power) \\
\hline SEPP 1 & On & Adjustable load: low, med, high \\
\hline SEPP 2 & Disabled & Cold redundancy \\
\hline Magnetorquer & Disabled & $X, Y$ and $Z$-axis \\
\hline CCSDS Engine & On & \\
\hline X-Band RX & Disabled & \\
\hline iADCS & Disabled & Fine ADCS \\
\hline S-Band TRX & On & RX only \\
\hline RWCAM board & On & Reaction wheel and HD-camera board \\
\hline HD-Camera & Disabled & \\
\hline Optical RX & Disabled & \\
\hline
\end{tabular}

feasible to cover a multitude of potential payload configurations within the scope of the TVAC test campaign, a representative use case was chosen. The corresponding payload units are shown in Table 1.

In terms of payloads that are enabled during the tests, a compromise had to be taken. It is desirable to use as many payloads as possible in order to provide comprehensive test coverage. However, the available power via the umbilical wire harness and battery capacity is limited. Additionally, it was decided to power off the fine ADCS due to a software problem at the time of test, that prevented reliable temperature readings. Of course, all components that were disabled during the TVAC tests have been tested in prior and subsequent unit level, subsystem level and system level tests.

Power generation The TVAC tests were performed in a thermally uniform environment, without any Sun simulator or other heat sources. Therefore, the only available power comes from the battery and from the umbilical wire harness. The umbilical harness is limited to a current of $1 \mathrm{~A}$ at $8 \mathrm{~V}$, which means that the chose use case will drain the battery eventually.

No radio transmission Both UHF and S-Band transmitter had to be switched off, to avoid damaging the respective receiver units due to reflections inside the TVAC chamber, that would exceed the maximum allowed input power of the receivers. In the particular case of the S-Band transmitter, this means that the additional 10-12 W of transmission power are not contributing to the heating of the spacecraft. As mentioned prior however, this turned out not to be significant as the S-Band transmitter is only operated during ground station passes. It is not contributing significantly to the spacecraft thermal behaviour, as became evident during the OPS-SAT commissioning phase. 


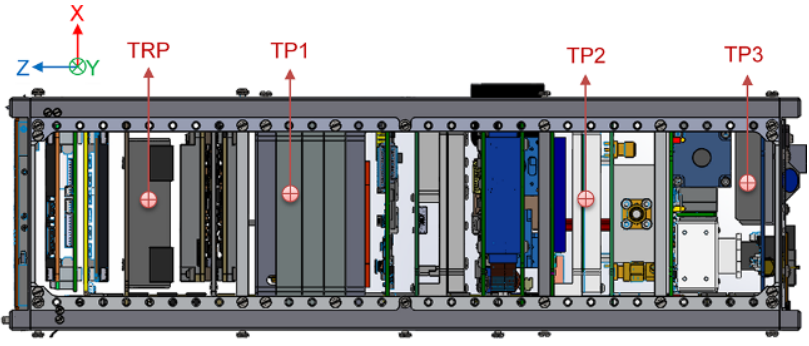

Fig. 3 Temperature sensors on the battery (TRP), SEPP (TP1), S-Band transceiver (TP2) and Optical receiver housing (TP3). These sensors are added for the purpose of testing, in order to monitor the respective unit's temperatures even when they are disabled

Time constraints TVAC tests naturally take a long amount of time, since the heat exchange between spacecraft and ambient (TVAC chamber) can only happen due to thermal radiation. Approaching a thermal plateau therefore can take anywhere from hours to days or even longer, depending on the size and thermal mass of the spacecraft. In the case of OPSSAT, the thermal test campaign was limited to four workdays, and the facilities could not be accessed during the night. To avoid starting from zero every day, the TVAC chamber was left in it's respective state at the end of the day and the tests were continued the next day. These constraints in effective test time meant, that only one full thermal cycle could be performed, as shown in the next section.

\section{Thermal sensors}

Aside from the multitude of temperature sensors that are integrated into the various bus and payload components of OPS-SAT, a variety of additional temperature sensors has been added for the TVAC tests. Those sensors can be further distinguished as internal temperature sensors and external temperature sensors. The internal sensors are mounted inside of the spacecraft body, directly on the corresponding payload components and can be found in Fig. 3 [3]. One sensor is placed on the battery (TRP), one on the SEPP housing (TP1), one on the S-Band transceiver (TP2) and one on the Optical receiver (TP3). The TRP sensor is critical as the battery has the lowest thermal limits.

The external sensors are shown in Fig. 4, with four sensors (TP4 to TP7) placed on the structure and one placed on the spacecraft front panel (TP8). Not shown in the figures are the sensors placed on the solar wings, the MGSE and the TVAC chamber [3]. Those sensor are listed in Table 2.

\section{Test setup and approach}

Satellite mounting In order to conduct tests in a thermal vacuum chamber, appropriate mounting of the spacecraft is required. It cannot simply be placed on

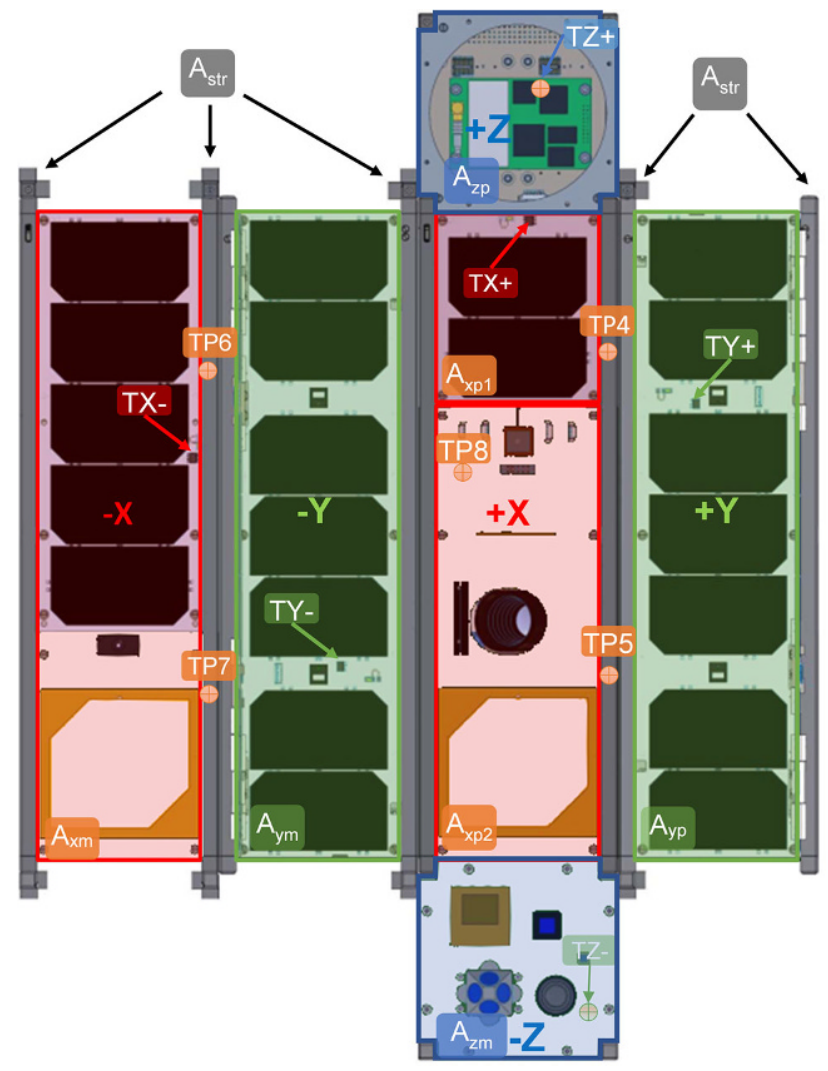

Fig. 4 Temperature sensors monitored durint TVAC tests, located on the outer surfaces of the spacecraft. The surfaces are folded flat onto a 2D plane for better overview. Identical colors indicated opposite faces of the three spacecraft axes $X($ red), $Y$ (green) and $Z$ (blue). The sensors TX+, TX-, TY+, TY-, $\mathrm{TZ}+$ and $\mathrm{TZ}-$ are integrated into the respective body panel. The temperature sensors TP4 to TP8 are additional sensors that have been mounted temporarily during the TVAC tests, to monitor the temperatures on the spacecraft structure

\begin{tabular}{|l|l|}
\hline Table 2 & Further external temperature sensors \\
\hline Sensor & Placement \\
\hline TP9 & Deployable solar wing, next to hinge \\
\hline TP10 & Bottom of MGSE \\
\hline TP11 & Top of MGSE \\
\hline TP12 & TVAC chamber processing plate \\
\hline TP13 & TVAC chamber shroud 1 \\
\hline TP14 & TVAC chamber shroud 2 \\
\hline
\end{tabular}

the bottom of the chamber, since this would lead to direct conductive heat exchange, which would not be representative of space environment. There are various ways to thermally decouple the spacecraft from the TVAC chamber, e.g. by suspending it with thin wires or strings or by using a mechanical mount with small contact area between chamber and spacecraft. A mechanical support, further referred to as Mechanical Ground Support Equipment (MGSE), was chosen for OPS-SAT due to to a lack of secure points for suspension. The contact points between spacecraft structure and MGSE are machined out of Teflon (PTFE). 


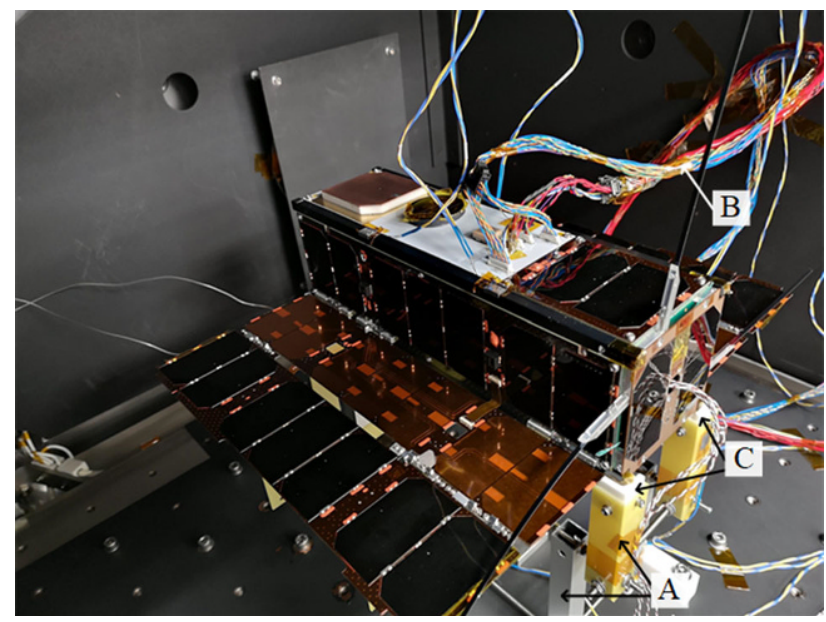

Fig. 5 OPS-SAT inside the TVAC chamber, resting on the MGSE $(A)$ and connected to its umbilical wire harness $(B)$ and the temperature sensors (not highlighted). The spacecraft structure is resting on PTFE elements mounted on top of the MGSE (white) on a small ciruclar surface of $5 \mathrm{~mm}$ diameter. The cut-out $90^{\circ}$ corners $(C)$ are for safety only, to prohibit the spacecraft from sliding out of the MGSE but are otherwise in no direct contact with the structure

PTFE was chosen because of its low thermal conductivity [6] on the one hand, but also because it was available in our facilities at the time and could easily be machined into the required shape.

Fig. 5 shows the satellite inside of the TVAC chamber [3], resting on the MGSE, as visible in the bottom right part of the figure (A). There are a total of four mechanical contact points (C), one on each corner of the spacecraft. Those contact points are reduced to a circular contact surface of $5 \mathrm{~mm}$ in diameter. While the mechanical and as such, the thermal interface between satellite and MGSE had been reduced as much as structurally feasible, a potential problem related to using an MGSE may become apparent. By placing a solid mechanical structure underneath the satellite, the thermo-optical view factor between the satellite and the TVAC chamber is reduced. Potential mitigation strategies for this problem may include: a different type of mechanical mount, i.e. suspension mount, reducing the physical dimensions of the MGSE to reduce view factor blocking or a surface treatment of the MGSE, in order to correlate with the thermo-optical properties of the TVAC chamber. The last point should be coupled with an adjustment of the dwell times at the thermal plateaus to consider the additional thermal mass of the MGSE.

Test phases One OPS-SAT TVAC test cycle can subdivided to seven distinct phases, that are briefly describe in the following list:

I Initial cool down: Bring the TVAC chamber and the satellite to temperature $T_{\text {start }}$, in order to start the temperature gradient determination phase

II Temperature gradient determination: Determination of the temperature gradient between the

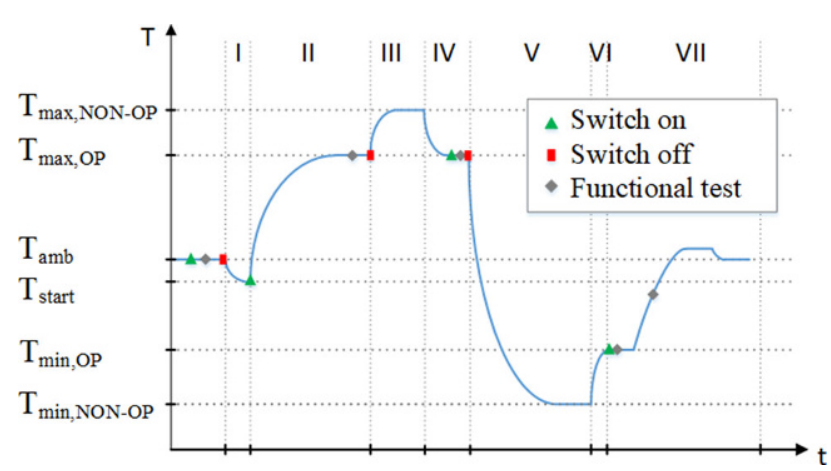

Fig. 6 TVAC test cycles, temperatures, spacecraft operational states and indicators for functional tests

critical subsystems and the TRP, as well as the temperature gradient between the TRP and the TVAC chamber. The satellite will be in an operational hot case at the end of this phase. This phase will provide useful information for subsequent temperature regulation of the TVAC chamber.

III Hot non-operational test: Expose the CubeSat to the uppermost survival temperature with respect to its most temperature sensitive component.

IV Hot operational test: Functional test after the hot non-operational phase. Possible to stop the test if failures are encountered.

V Cold non-operational test: Expose the CubeSat to the lowest survival temperature with respect to its most temperature sensitive component.

VI Cold start-up phase: Increase temperature to cold switch-on temperature and switch on the respective CubeSat components. Monitor the battery heater. Perform functional tests. Possible to stop the test if failures are encountered.

VII Final ambient plateau \& re-pressurisation: Bring TVAC chamber back to a few degrees above ambient temperature to avoid condensation. Then pressurize the TVAC chamber.

A graphical representation of the TVAC test timeline is shown in Fig. 6, indicating the respective phase, corresponding temperature and state of the spacecraft [3].

Initial phase Phases I and II, the initial cool down and the temperature gradient determination phase are a crucial part of the test that serves the purpose of identifying the temperature difference, $\Delta T$, between the spacecraft in a thermal equilibrium state and the TVAC chamber. This ensures that the spacecraft does not eventually exceed any of the component's maximum allowable operating temperature at a given ambient temperature and a given power consumption. If the expected component temperatures are known, e.g. due to thermal simulation, the TVAC chamber can be set to the corresponding temperature at start. Otherwise, it is a best guess scenario, that may require a couple of iterations if the initial temperature of the TVAC chamber is set too high. On the other hand, it takes more time for initial cooling of the chamber 
and the spacecraft, if the initial temperature is chosen too low. For OPS-SAT, the initial temperature of the TVAC chamber was chosen at $5^{\circ} \mathrm{C}$, which turned out to be a good guess as this led to a final battery temperature just below $42^{\circ} \mathrm{C}$, very close to the maximum allowable $45^{\circ}$. In other words: by increasing the TVAC chamber temperature by another $3^{\circ} \mathrm{C}$, the maximum operational temperature of the battery can be reached.

Test cycle A test cycle can be summarised by the phases II to VI, as shown in Fig. 5. Typically, a couple of those cycles should be performed in the course of a TVAC test campaign, however, due to time constraints, only one full cycle could be conducted. As the corresponding temperatures suggest, the spacecraft is brought to it's maximum operational temperature first (phase II) and kept at this temperature until a predefined amount of time, the so-called dwell time, has elapsed. Note that after the first full cycle, no more temperature gradient has to be determined during phase II. In phase III, the spacecraft is powered down and brought to its maximum non-operational temperature. Since the spacecraft is now not producing any heat on its own, this has to be achieved by increasing the temperature of the TVAC chamber accordingly. After another dwell time period has elapsed, the spacecraft is brought down once more to maximum operational temperature (phase IV) and a defined set of functional tests is executed, before the spaceraft is powered down again and cooled to its minimum non-operational temperature (phase V). In phase VI, the temperature is increased towards the minimum operational temperature of the spacecraft and a coldstart is performed, followed by functional checks.

Final phase Once one or more test cycles are complete, the TVAC chamber is brought back up to a few degrees above ambient temperature, to avoid any condensation on the spacecraft. The spacecraft is kept at an operational state during this temperature increase, until its maximum allowable temperature is reached. Functional tests are performed throughout the whole period. Finally, the TVAC chamber is pressurized again.

Safety margin A safety margin of $1^{\circ} \mathrm{C}$ was subtracted from all maximum and minimum operational and non-operational temperatures to account for sensor inaccuracies and uncertainties in temperature regulation.

\section{Results}

This section shows the results of the OPS-SAT TVAC test campaign with respect to the additional temperature sensors that have been placed at key components inside of the spacecraft on the outside of the spacecraft. The temperature sensors that are integrated into the various bus and payload units are not shown, as this would exceed the scope of this article.

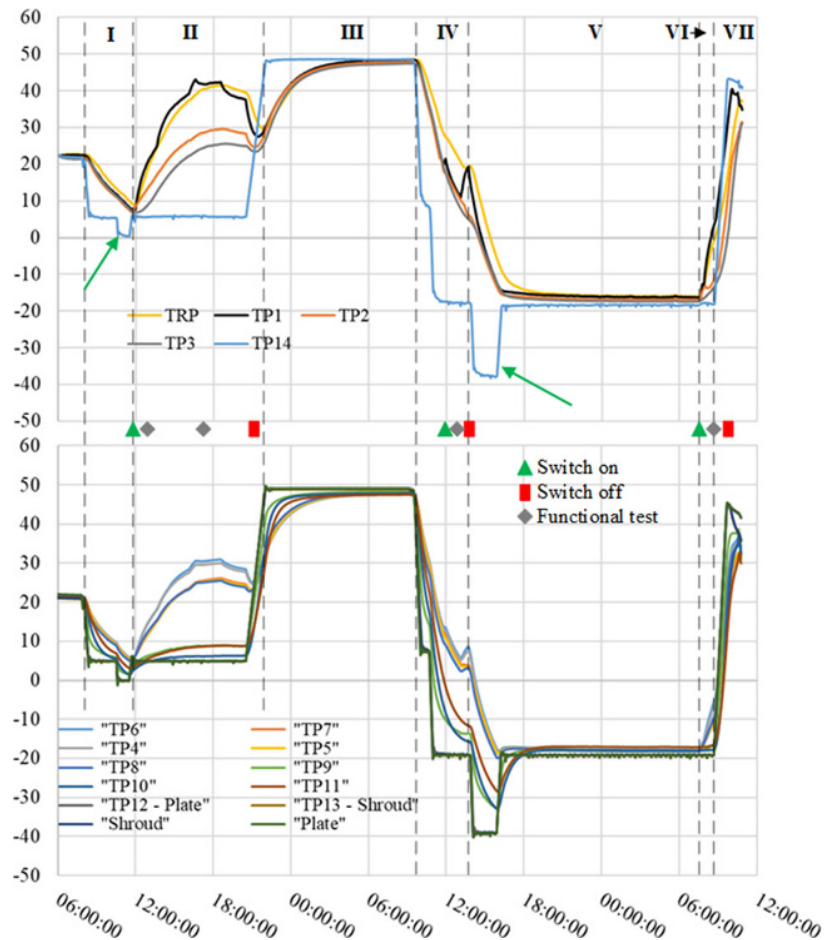

Fig. 7 TVAC test campaign thermal sensor measurements. The top sections shows the internal sensors: TRP (battery), TP1 (SEPP), TP2 (S-Band transceiver), TP3 (Optical Receiver) and TP14 (TVAC chamber shroud). The different phases of the test are indicated as roman numerals at the top. The bottom section shows the external sensors: TP4-7 (structure), TP8 (front body panel), TP9 (solar wing), TP10-11 (MGSE), TP1213 (TVAC chamber). Power states of the spacecraft are shown as green and red markers and the start of functional tests is indicated as grey marker

Internal sensors The top graph of Fig. 7 shows the test phases I to VII and the corresponding temperatures for the additional internal temperature sensors which are highlighted in Fig. 3. The OPS-SAT battery acts as temperature reference point TRP and the temperatures are shown additionally for the SEPP (TP1), the S-Band transceiver (TP2), the Optical receiver (TP3) and the thermal chamber shroud [3].

The test starts at ambient temperature at the section prior to phase I followed by a cool-down to $5^{\circ} \mathrm{C}$ before powering on the spacecraft. Power states and and functional tests are highlighted as green triangle (power on), red rectangle (power off) and grey diamond (functional test). The TVAC chamber temperature (blue curve, TP14) is decreased on purpose two times (green arrows) to speed up the respective cooling phases of the spacecraft. The changes in slope of the SEPP temperature (black curve, TP1) during phase III serve the purpose of accelerating the heating of the spacecraft by setting the SEPP to a high power consuming state. The TVAC chamber dictates the overall temperatures during the non-operational phases, as nicely visible shown in phase III and V. All temperatures follow the TVAC chamber in the non- 
operational case but do not fully approach this temperature.

The relation between the battery temperature (TRP) and the rest of the powered payloads and components is most critical for OPS-SAT, since the battery is most critical from a thermal point of view. The results show, that the battery is in close relation to the temperature on the SEPP (TP1). This behaviour can be related to the physical proximity of the SEPP and the comparably large power draw of the SEPP, compared to the rest of the powered payloads. It is further clear form TP2 and TP3, that the temperatures of the less power consuming payloads are significantly lower and appear to gradually decrease with increasing distance from the SEPP.

External Sensors The bottom graph of Fig. 7 shows the temperatures of the external sensors placed on the spacecraft structure, as shown in Fig. 4. The sensors TP4 and TP5 are placed on the front side of the structure and TP6 and TP7 are placed on the backside of the structure. TP8 is placed on the front body panel. TP9 is placed on a deployable solar wing, just next to its hinge. TP10 and TP11 are placed on the bottom and the top of the MGSE repsectivel and TP12 to TP14 are placed at various locations inside the TVAC chamber. Most noteworthy with respect to the external sensors, is that the structure and body mounted sensors TP4 to TP8 follow the SEPP temperatures in close relation, albeit at roughly $10^{\circ} \mathrm{C}$ difference. A temperature gradient can be observed with increasing distance from the SEPP, leading to higher temperatures on the sensors in closer proximity to the SEPP, namely TP4 and TP6. Further noteworthy is the behaviour of TP11 and TP12, both in very close range, well below the temperatures of the active components but still roughly $4^{\circ} \mathrm{C}$ above the TVAC chamber itself. For TP12, this means that there is some remaining heat transfer between the solar wings and the spacecraft structure via the solar wing hinges. For TP11, this means that there is some remaining heat transfer between the spacecraft structure and the top part of the MGSE. TP10 can be seen at rougly $1^{\circ} \mathrm{C}$ above TVAC chamber temperature, meaning that there is conductive heat transfer between the spacecraft and the TVAC chamber via the MGSE.

Functional tests The predefined set of functional tests was executed successfully during all phases and all temperatures. An additional set of tests was performed subsequent to the TVAC tests, in order to verify that no late effects have occurred.

\section{Conclusion}

Summary OPS-SAT is a versatile and, for its size, powerful spacecraft. This versatility comes at the cost of testing complexity throughout all test-campaigns, including the TVAC test campaign. Several compromises had to be made to conduct the TVAC tests within the given time frame of four days. This includes selection of a subset of payloads that can be switched on during the tests. On the one hand, not every possible constellation that is required by an OPS-SAT experiment can be tested, on the other hand a subset of payloads had to be selected due to charging power constraints. The radio transmitters could only be powered on in receive mode, leading to significantly less power draw which is mitigated through the fact, that radio transmissions are not continuous and only active for a couple of minutes during ground station passes. The time frame for the tests only allowed for one full cycle of thermal vacuum tests. To mitigate this problem, the time frame could be extended or alternatively, night time could be used additionally for active testing.

TVAC tests The TVAC test results show a strong correlation between the SEPP temperature and the OPSSAT battery, leading to the conclusion that the SEPP is a major factor to consider, when running OPS-SAT experiments for extended time periods. A negative temperature gradient is observed with increasing distance from the SEPP. This is to be expected, as the SEPP is the most prominent heat source during the tests. The marginal temperature changes on the solar wings leads to the conclusion, that the wings are in poor thermal contact with the rest of the spacecraft. The wings, therefore, can only contribute marginally for cooling or heating, depending on the respective attitude in orbit, i.e. whether a wing is currently illuminated by the Sun or facing cold space. The temperature sensors on the MGSE show, that the chosen MGSE design is not ideal as the MGSE is not fully thermally decoupled from the spacecraft. This leads to a marginal thermally conductive link between MGSE and TVAC chamber, that is in the same order of magnitude as the thermal link between spacecraft and solar wings.

Functional tests The most important conclusion from the TVAC tests is, of course, that the functional tests could be executed successfully during all phases. This shows, that OPS-SAT and its components can be successfully operated under vacuum conditions and under the thermal limits of the individual components. It shall be noted at this point, that all payloads that were not switched on during the TVAC test campaign have been validated in the respective preceding unit and subsystem tests under vacuum conditions and thermal limit conditions.

Lessons learned Improvements for future test campaigns will be based on the lessons learned during the OPS-SAT TVAC tests and preparation. A critical point was the selection of powered payload components during the respective hot and cold operational tests and the corresponding functional tests. Ideally, all components should be included in future tests, and this requires appropriate planning, as not all components may be powered simultaneously. Addition of a Sun simulator would yield a more realistic on-orbit scenario, rather than a uniform temperature environ- 
ment and might reveal attitude-dependent thermal behaviour that could not be observed with a uniform environment.

Planning and construction of the MGSE should be considered well in advance of the test campaign and in coordination with the available TVAC facilities. The mechanical interface between MGSE should be planned with care and as little contact surface as possible. Additionally, any blocking of the view factor between spacecraft and TVAC chamber needs to be considered, in particular if a Sun simulator is used, to avoid any shading of the solar arrays.

Finally, sufficient time and margin is important for such a test. In particular the time required for the spacecraft to reach the respective hot and cold plateaus is unknown prior to the tests.

Funding Open access funding provided by Graz University of Technology.

Open Access This article is licensed under a Creative Commons Attribution 4.0 International License, which permits use, sharing, adaptation, distribution and reproduction in any medium or format, as long as you give appropriate credit to the original author(s) and the source, provide a link to the Creative Commons licence, and indicate if changes were made. The images or other third party material in this article are included in the article's Creative Commons licence, unless indicated otherwise in a credit line to the material. If material is not included in the article's Creative Commons licence and your intended use is not permitted by statutory regulation or exceeds the permitted use, you will need to obtain permission directly from the copyright holder. To view a copy of this licence, visit http://creativecommons.org/licenses/by/4.0/.

\section{References}

1. Evans DJ (2016) OPS-SAT: operational concept for ESA'S first mission dedicated to operational technology. In: SpaceOps 2016 Conference. American Institute of Aeronautics and Astronautics, https://doi.org/10.2514/6.20162354

2. Binder M, Finsterbusch R, Henkel M, Patrick R, Teschl F, Unterberger M, Zeif R, Hörmer AJ, Kubicka M (2019) OPSSAT system design report. Issue 4.4. Graz University of Technology,

3. Kubicka M, Zeif R, Koudelka O (2021) OPS-SAT thermal vacuum test report. OPSSAT-AIV-TVAC-TR_v1.7, Issue 1.7. Graz University of Technology,

4. Kubicka M (2021) Thermal modelling and on-orbit sensor calibration and analysis on ESA's OPS-SAT nanosatellite. Graz University of Technology,

5. Kubicka M, Koudelka O, Evans D, Zeif R, Henkel M, Hörmer AJ (2020) Thermal vacuum tests and thermal properties on ESA's OPS-SAT mission. In: 2020 International Conference on Broadband Communications for Next Generation Networks and Multimedia Applications (CoBCom), pp 1-7 https://doi.org/10.1109/CoBCom49975.2020.9174095

6. Buerkle M, Asai Y (2017) Thermal conductance of teflon and polyethylene: insight from an atomistic, single-molecule level. Sci Rep 7:1. https://doi.org/10.1038/srep41898

7. Zeif Reinhard, Henkel M, Hörmer AJ, Kubicka M, Wenger M, Koudelka O (2018) The redundancy and fail-safe concept of the OPS-SAT payload processing platform. In: 69th Inter- national Astronautical Congress IAC 2018, 01-05.10.2018

(http://www.iac2018.org)

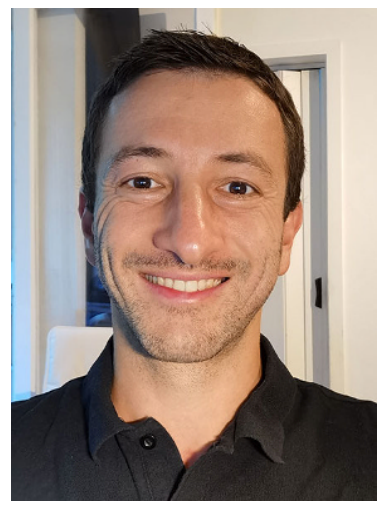

Manuel Kubicka, studied Space Sciences and Earth from Space at Graz University of Technology, with a graduation in 2016. After that, he started a PhD Research position on ESA's OPSSAT project, under an ESA Networking Partnering Initiative (NPI) contract, starting at theEuropean Space Operations Centre (ESOC) in Darmstadt Germany. This work was continued in 2017 at Graz University of Technology with a focus on testing, thermal modelling and on-orbit sensor evaluation on OPS-SAT.

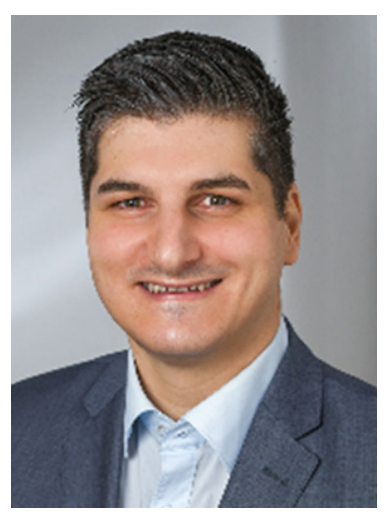

Reinhard Zeif, studied Telematics at the Graz University of Technology and graduated in 2011. Afterwards, he worked in the automotive chip industry. Since 2014, he has been employed as research engineer at the Institute of Communication Networks and Satellite Communications at the Graz University of Technology. Mr Zeif is the responsible systems engineer for the OPS-SAT mission.

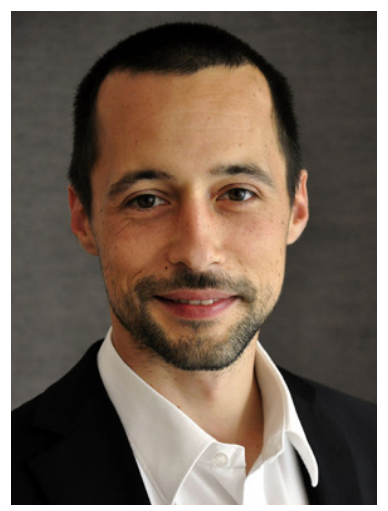

Maximilian Henkel, studied Telematics (Information and Communication Technology) at Graz University of Technology with focus on Control Systems and Software Technology. He graduated in 2015 and has since then been working as research engineer at the Institute of Communication Systems and Satellite Communications as member of the OPS-SAT team. 


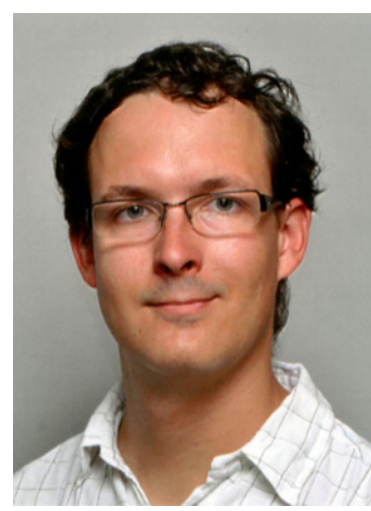

Andreas Hörmer, studied Telematics at the Graz University of Technology with focus on Telecommunications and Audio Technology and graduated in 2017. Since then, he has been working as research engineer at the Institute of Communication Systems and Satellite Communications at Graz University of Technology. He was project member in the OPS-SAT project and is the systems engineer for the PRETTY satellite. 